\title{
EIGENVALUES OF THE LAPLACIAN FOR RECTILINEAR REGIONS
}

\author{
H. P. W. GOTTLIEB ${ }^{1}$
}

(Received 16 June 1986; revised 18 December 1986)

\begin{abstract}
From a knowledge of the eigenvalue spectrum of the Laplacian on a domain, one may extract information on the geometry and boundary conditions by analysing the asymptotic expansion of a spectral function. Explicit calculations are performed for isosceles right-angle triangles with Dirichlet or Neumann boundary conditions, yielding in particular the corner angle terms. In three dimensions, right prisms are dealt with, including the solid vertex terms.
\end{abstract}

\section{Introduction}

Information about the geometry of a domain and about the nature of the boundary conditions is yielded through the asymptotic expansion of the spectral function

$$
\sum_{n} \exp \left(-\lambda_{n} t\right), \quad t \rightarrow 0
$$

where $\lambda_{n}$ are the eigenvalues of the Laplacian

$$
\left(\nabla^{2}+\lambda\right) \psi=0
$$

The general theory of this subject has been developed by Pleijel [9], Kac [6], McKean and Singer [7], Waechter [15], Sleeman [10] and others by regarding (1.2) as arising from the heat (diffusion) equation and consideration of the appropriate Green's functions.

\footnotetext{
${ }^{1}$ School of Science, Griffith University, Nathan, Queensland 4111, Australia.

(C) Copyright Australian Mathematical Society 1988, Serial-fee code 0334-2700/88
} 
The study of explicit examples such as those to be listed below is especially important because of the light they may shed on the uniqueness problem, i.e. whether equality of spectra implies geometrical congruence of the corresponding domains. For dimensions four and higher, Urakawa [14] has shown that the inverse problem does not have a unique solution; the problem is still open in two or three dimensions. The higher-dimensional examples presented in [14] have boundaries which are not smooth but are polygons, suggesting that rectilinear regions in two and three dimensions warrant close investigation.

The present paper is concerned in particular with right-angled isosceles triangles with Dirichlet or Neumann boundary conditions, and with triangular right prisms, as well as with more general convex polygonal right prisms. Special attention is paid to corner angle terms (two dimensions) and solid vertex terms (three dimensions); an expression for the contribution of a general right solid angle is derived.

Over the past few years, explicit demonstrations of the geometrical characteristics obtained from (1.1) have been presented for a variety of specific regions, by Stewartson and Waechter [13], Waechter [15], Zayed [16], [17], Sleeman and Zayed [12], and the author [3], [4]. In the list which follows, $D$ represents Dirichlet and $N$ represents Neumann boundary conditions.

ONE-DIMENSIONAL. Straight line: [16] $D \& N$. (Zayed's work for the line, and also for the rectangle and cuboid (see below), actually deals with a linear combination of $D$ and $N$ at either end.)

TWO-DIMENSIONAL.

Rectangle: [16] $D \& N$; [12] $D, N$; [4] $D \& N$.

Circle: [13] D.

Circular annulus: Limit as outer radius $\rightarrow \infty:[13] D$.

Thin: [3] $D ;$ [4] $N$,

Thick: [12] $D \& N$.

Annular sector: Thin: [4] $D \& N$.

THREE-DIMENSIONAL.

Cuboid: [15] $D$; [16] $D \& N$; [12] $D, N$; [4] $D \& N$.

Cylinder: (finite): [15] $D$; [12] $D, N$.

Annular cylinder (finite): Thin: [4] $D \& N$.

Thick: [12] $D, N$.

Sphere: [15] $D$.

Spherical shell: Thin: [4] $D, N$.

Thick: [17] $D \& N$.

References [13], [15], [16], [12], [17] performed their calculations via Green's functions for the heat equation and Laplace transforms, etc. By contrast, our method [3], [4] proceeds via the wave equation and direct use of the explicit 
eigenvalues of (1.2) and their analytical properties; the Poisson summation formula is the main summation tool in (1.1).

It is important to extend this list of specific calculations. Investigations such as those listed above and the present paper may be considered to serve a three-fold purpose. They give concrete illustrations of the various features and results of general theorems which they verify by way of explicit realizations; they provide a wider class of examples which iend additional force to conjectures not yet proved in their generality; and they assist in extending conjectures by suggesting the forms which further terms in a general expansion theorem might take.

According to references [6], [7], [13] (Dirichlet boundary conditions) and [9], [10], [4] (Neumann boundary conditions), the spectral function (1.1) in two dimensions for convex polygonal domains has the asymptotic expansion

$$
E_{2} \sim \frac{A}{4 \pi t}+\frac{L_{N}-L_{D}}{8(\pi t)^{1 / 2}}+\left(\sum_{D D, N N}-\sum_{D N}\right) \alpha(\theta)+\cdots, \quad t \rightarrow 0,
$$

where $A$ is the area of the domain, $L_{D}\left(L_{N}\right)$ is the length of the part of the boundary with Dirichlet (Neumann) boundary condition, and the summation is over angles at corners subtended by pairs of adjacent sides having the indicated boundary conditions. The corner angle term $\alpha$, for angle $\theta$, is given by [7]

$$
\alpha(\theta)=\frac{\pi^{2}-\theta^{2}}{24 \pi \theta} .
$$

(Actually, if the boundary of the domain is too rough ("fractal domain"), even the second term in (1.3) may have to be modified as to the definition of the measure: see [2].)

In three dimensions, the work of Waechter [15] for Dirichlet boundary condition (first three terms) and of the author [4] for Neumann boundary condition indicates that, for a convex region bounded by planes, the asymptotic expansion of $(1.1)$ reads

$$
\begin{aligned}
E_{3}- & \frac{V}{8(\pi t)^{3 / 2}}+\frac{S_{N}-S_{D}}{16 \pi t}+\frac{1}{2(\pi t)^{1 / 2}}\left(\sum_{D D, N N}-\sum_{D N}\right) \alpha(\theta) L(\theta) \\
& +\left(\sum_{N N N, D D N}-\sum_{D D D, D N N}\right) \chi(\Omega)+\cdots, \quad t \rightarrow 0,
\end{aligned}
$$

where $V$ is the volume of the region and $S_{D}\left(S_{N}\right)$ is the total area of those surfaces with Dirichlet (Neumann) boundary condition. $L(\theta)$ is the length of those edges formed by two adjoined faces which make an angle of $\theta$ with each other and which have the pairwise combination of boundary conditions indicated in the summations.

The question of vertices was left open by Waechter [15]. The constant term in (1.5), due to vertices, was conjectured in [4] to have the given summation form where the three labels denote the boundary conditions on the three faces 
subtending the solid angle $\Omega$ at a vertex. It was shown in [4] that for a solid angle of $4 \pi / 8$, the vertex contribution is $\chi(\pi / 2)=1 / 64$. The general expression for the vertex function $\chi(\Omega)$ is not known; we give a partial answer below.

In this paper, we use the direct method of [4] to perform first of all the calculation of the asymptotic expansion of the spectral function (1.1) for the case of a right-angled isosceles triangle with Dirichlet or Neumann boundary conditions. This example is particularly interesting because it involves corner angles which are not all right angles.

In three dimensions, we calculate the expansion for a prism with the above triangular cross-section. Then we calculate for a general right prism on a convex polygonal base, assuming the expansion for the latter is known in two dimensions. For the case of any right solid angle, that is, a solid angle bounded by three planes, two of which are perpendicular to the third, we show in Section 4 that the general expression for the vertex function $\chi$ is given in terms of the corner angle term $\alpha$ (see (1.4) above) by

$$
\chi(\Omega)=\alpha(\Omega) / 4 \text {. }
$$

The explicit value $\chi(\pi / 4)=5 / 128$ is verified in Section 3.1 .

A limiting procedure applied to the expressions (1.4) and (1.6) above is used in Appendix A to recover the simple constant numerical terms in the expansions for a circle and a right circular cylinder. Some summation formulae used throughout this paper are presented in Appendix B.

\section{Isosceles right-angle triangle}

\subsection{Dirichlet boundary condition}

The complete set of independent eigenfunctions for vibrations of an isosceles right-angle triangular membrane (with fixed sides $0 \leqslant x \leqslant a, 0 \leqslant y \leqslant a, y=a$ $-x)$ is given by [8] (p. 756) as

$$
\begin{aligned}
\psi_{m, n}^{(D)}(x, y)= & \sin [(m+n) \pi x / a] \sin [n \pi y / a] \\
-(-1)^{m} \sin [(m+n) \pi y / a] & \sin [n \pi x / a] ; \\
& m, n=1,2,3, \ldots,
\end{aligned}
$$

with corresponding eigenvalues

$$
\lambda=k_{m n}^{2}=(\pi / a)^{2}\left[(m+n)^{2}+n^{2}\right] .
$$

The spectral function (1.1) for this triangle with Dirichlet boundary condition is

$$
E_{2}^{(D)}=\sum_{m=1}^{\infty} \sum_{n=1}^{\infty} \exp \left\{-\left(\pi^{2} t / a^{2}\right)\left[(m+n)^{2}+n^{2}\right]\right\}
$$


The asymptotic expansion of the right hand side of (2.3) is found explicitly by utilizing the Poisson summation formula result (as in [4])

$$
\sum_{m=0}^{\infty} \exp \left(-z m^{2}\right)-\sqrt{\pi} /(2 \sqrt{z})+\frac{1}{2}, \quad z \rightarrow 0 .
$$

The double sum in (2.3) is converted to a form in which (2.4) may be invoked by using the summation formula (B.5) of Appcndix $\mathrm{B}$ with $f(m, n)=$ $\exp \left\{-\left(\pi^{2} t / a^{2}\right)\left[m^{2}+n^{2}\right]\right\}$. The second and third terms in (B.5) then correspond to $z=2 \pi^{2} t / a^{2}$ and $z=\pi^{2} t / a^{2}$ respectively in (2.4), whilst the exponential in the first term may be factorized to untangle the $m$ and $n$ dependence and gives the product of two terms of the form (2.4). In this manner we obtain

$$
E_{2}^{(D)} \sim \frac{\left(a^{2} / 2\right)}{4 \pi t}-\frac{(2+\sqrt{2}) a}{8(\pi t)^{1 / 2}}+\frac{3}{8}, \quad t \rightarrow 0
$$

with only exponentially small errors (see [7], p. 67). For our triangle, with area $A=a^{2} / 2$ and total perimeter (with Dirichlet boundary condition)

$$
L_{2}=2 a+\sqrt{2} a,
$$

the expansion (2.5) precisely mimics, i.e. verifies, the general form (1.3), since by (1.4)

$$
\alpha\left(\frac{\pi}{2}\right)=\frac{1}{16} ; \quad \alpha\left(\frac{\pi}{4}\right)=\frac{5}{32}
$$

and $1 / 16+2(5 / 32)=3 / 8$ giving the constant term as in (2.5).

This result is significant in that it verifies explicitly the corner angle term (1.4) for the case of angle $\pi / 4,(2.7 \mathrm{~b})$. This does not seem to have been elicited before for an angle not equal to $\pi / 2$.

\subsection{Neumann boundary condition}

The eigenfunctions in this case are as in (2.1) but with cosine functions and a relative plus sign. The eigenvalues are again given by (2.2), but with $m, n=$ $0,1,2, \ldots$ The summation formula (B.4) is now used in the spectral function (1.1) and we obtain (2.5) again but with a change of sign for the second term, to a plus. Thus the general form (1.3) is verified for this configuration with Neumann boundary condition along the perimeter.

\section{Isosceles right-angle triangular right prism}

In this section we consider the three-dimensional case of a triangular right prism of height $b, 0 \leqslant z \leqslant b$, standing on the isosceles right-angle triangular base of the previous section. We evaluate the spectral sum (1.1) to elicit volume, 
surface area, perimeter, etc., in the cases of Dirichlet or Neumann boundary condition on the sides, with either Dirichlet or Neumann boundary condition on the top and bottom faces.

\subsection{Dirichlet condition on sides}

\subsubsection{Dirichlet condition on base and top}

The relevant solution to the Helmholtz equation (1.2) is

$$
\Psi_{l m n}^{(D)}(x, y, z)=\sin [l \pi z / b] \psi_{m n}^{(D)}(x, y) ; \quad l=1,2, \ldots
$$

with $\psi_{m n}^{(D)}$ given by (2.1). Now

$$
\lambda=\left(\pi^{2} / b^{2}\right) l^{2}+k_{m n}^{2}
$$

with $k_{m n}^{2}$ given by (2.2).

The spectral function (1.1) is given by a triple sum which factorizes into the form

$$
E_{3}^{(D)}=\left[\sum_{l=1}^{\infty} \exp \left\{-\left(\pi^{2} t / b^{2}\right) l^{2}\right\}\right] E_{2}^{(D)}
$$

with $E_{2}^{(D)}$ given by (2.3). This may be evaluated explicitly in the asymptotic limit as $t \rightarrow 0$ by using (2.4) and (2.5), from which we obtain

$$
E_{3}^{(D)} \sim \frac{A b}{8(\pi t)^{3 / 2}}-\frac{2 A+b L_{2}}{16 \pi t}+\frac{2 L_{2}+6 b}{16\left[2(\pi t)^{1 / 2}\right]}-\frac{3}{16},
$$

again with only exponentially small errors.

Our prism has volume $V=A b$ and surface area $S=2 A+b L_{2}$ (see (2.6)). Edges of total length $2 L_{2}+b$ have adjacent sides making a constant angle of $\pi / 2$, with corner angle factor $1 / 16$ (see (2.7a)), and two edges of length $b$ each have adjacent sides making a constant angle of $\pi / 4$, with corner angle factor $5 / 32$ (see (2.7b)), so in (1.5),

$$
\sum L(\theta) \alpha(\theta)=2 L_{2} / 16+b[1 / 16+2(5 / 32)] .
$$

This sum (3.5) over all edges, as the coefficient of $\left[2(\pi t)^{1 / 2}\right]^{-1}$ in (1.5), is thus yielded explicitly by the third term in (3.4).

The expansion (3.4) therefore precisely verifies the general three-dimensional form (1.5) for Dirichlet boundary conditions for its first three terms. We now use the fourth, constant, term in (3.4) to evaluate a vertex term in the corresponding expression in (1.5). There are two solid angles of $4 \pi / 8=\pi / 2$ steradians within our prism, each with a vertex factor known, from [4], to be

$$
\chi(\pi / 2)=\frac{1}{64} \text {. }
$$


There are four solid angles of $4 \pi / 16=\pi / 4$ steradians. Hence we must have $2(1 / 64)+4 \chi(\pi / 4)=3 / 16$, whence we deduce that

$$
\chi(\pi / 4)=\frac{5}{128} \text {. }
$$

The term (3.7) now provides another value, along with (3.6), against which any general differential geometric theory would have to check its constant term in the asymptotic expansion of (1.1), In Section 4 we shall find an expression which covers more general solid angles.

\subsubsection{Neumann condition on base and top}

Here,

$$
\Psi_{l m n}(x, y, z)=\cos [l \pi z / b] \psi_{m n}^{(D)}(x, y) ; \quad l=0,1,2, \ldots
$$

Using previous methods, calculation yields explicitly an expression containing the individual terms as in (3.4) but with all the appropriate sign changes corresponding to the fact that certain of the faces have Neumann boundary condition and this also categorizes the edges and vertices accordingly. Thus the form of (1.5) in this configuration is also precisely verified.

\subsubsection{Dirichlet condition on base, Neumann condition to top}

Here,

$$
\Psi_{l m n}(x, y, z)=\sin [(l+1 / 2) \pi z / b] \psi_{m n}^{(D)}(x, y) ; \quad l=0,1,2, \ldots
$$

and

$$
\lambda=\left(\pi^{2} / b^{2}\right)\left(l+\frac{1}{2}\right)^{2}+k_{m n}^{2} .
$$

As was done for (3.3), $E_{3}$ may be factorized into one- and two-dimensional parts. The asymptotic form for the first factor is obtained from equation (B.7) of Appendix B of reference [4] which dealt with Poisson summation formulae; equation (2.5) for $E_{2}$ is used for the second factor. We calculate

$$
E_{3}-\frac{A b}{8(\pi t)^{3 / 2}}-\frac{b L_{2}}{16 \pi t}+\frac{3 b}{8} \frac{1}{2(\pi t)^{1 / 2}} .
$$

This again verifies (1.5), taking into account all sign changes and any consequent cancellations due to the combinations of Dirichlet and Neumann conditions.

\subsection{Neumann condition on sides}

For these cases, the formalism of Section 3.1 follows through, except that $\psi(x, y)$ and $E_{2}$ are as described in Section 2.2. Calculations for each of the cases:

\subsubsection{Dirichlet condition on base and top;}




\subsubsection{Neumann condition on base and top;}

\subsubsection{Dirichlet condition on base, Neumann condition on top;}

do verify explicitly the form (1.5), with the sign changes and cancellations in the various terms arising in a similar manner to those described in Section 3.1 above.

\section{Convex polygonal right prisms}

The previous examples suggest that, given a two-dimensional result, the corresponding results for a three-dimensional right prism of that cross-section will automatically follow. This may be proved as follows.

For a domain in the $x-y$ plane with convex polygonal boundary, along each straight line of which there may be either Dirichlet or Neumann boundary condition, the two-dimensional spectral function is assumed to be of the form (1.3), with corrections which are exponentially small or at most $o(\sqrt{t})$. Then the three-dimensional spectral function for the corresponding right prismatic case is just the product of the two-dimensional spectral function mentioned above and the one-dimensional spectral function corresponding to the straight line as in Section 3 with appropriate end conditions.

This procedure is akin to that of Waechter ([15], Section 4), which dealt with Dirichlet boundary condition only, and of [11], both of which assumed a simply-connected domain, and is also described for a particular multiplyconnected case (annular cylinder) in [4], Section 4.2; these references all dealt with smooth curved boundary of cross-section.

Using Poisson summation formulae such as (2.4), it is in fact easy to show first of all that the one-dimensional spectral function for the straight line $0 \leqslant z \leqslant b$ has the asymptotic expansion

$$
E_{1} \sim \frac{b}{2(\pi t)^{1 / 2}}+\left(\sum_{N}-\sum_{D}\right) \frac{1}{4}, \quad t \rightarrow 0
$$

the sum being over ends with the indicated boundary condition. Forms of equation (4.1) and its extension to mixed boundary conditions have been obtained by a lengthy procedure via Green's functions in [16]. (The errors in (4.1), declared in [16] to be $O\left(t^{1 / 2}\right)$, are actually determined by our above procedure to be exponentially small.) Thus "one can hear the length of a uniform string" (c.f. [6]) even if the end conditions are unknown, and one may distinguish between those end conditions.

Now, using the product of (1.3) for the cross-sectional region and (4.1) as explained above, we obtain, for the asymptotic expansion of the three-dimensional spectral function for the corresponding right prism, an explicit form whose 
first three terms, after interpretation of the geometrical meanings of the various symbols involved, precisely verifies the form of those in (1.5) for this geometrical configuration. The fourth, constant, term obtained in the above manner is

$$
\left(\sum_{N}-\sum_{D}\right)\left(\sum_{D D, N N}-\sum_{D N}\right) \alpha(\theta) / 4=\left(\sum_{N N N, D D N}-\sum_{D D D, D N N}\right) \alpha(\theta) / 4 .(4.2)
$$

From this resulting expression we are able to identify and evaluate the general expression for the vertex function $\chi$ in (1.5) for any right solid angle, i.e. any solid angle bounded by three planes, two of which are perpendicular to the third. If the first two planes make an angle of $\theta$ radians with each other, then the solid angle subtended at the vertex by the three faces is $\Omega=(4 \pi / 4) /(\pi / \theta)=\theta$ steradians. For these configurations we obtain, from comparison of the fourth term in (1.5) with (4.2), the formula

$$
\chi(\Omega \text { sterad. })=\frac{1}{4} \alpha(\Omega \mathrm{rad} .),
$$

where the function $\alpha$ has been defined in (1.4).

A form of expression (4.2), for the case of Dirichlet condition only or Neumann condition only, was obtained via a Green's function approach by Baltes [1]. Equation (4.3) in (1.5) is a general expression for a right solid vertex. It was verified for $\Omega=4 \pi / 8=\pi / 2$ in [4] (by (2.7a) and (3.6) above), and has now been verified in the present paper for $\Omega=4 \pi / 16=\pi / 4$, by (2.7b) and (3.7) above.

\section{Conclusion}

The calculations in this paper have displayed explicitly various geometrical and boundary-condition-dependent terms in the asymptotic expansions of the spectral function (1.1) for further two- and three-dimensional rectilinear regions with Dirichlet and Neumann boundary conditions. In particular, in two dimensions the corner angle term (1.4) was elicited in (2.7b) for the angle $\theta=\pi / 4$ by dealing with an isosceles right-angle triangle, and in three dimensions the corresponding vertex term in a right prism was evaluated in (3.7) for the solid angle $\Omega=\pi / 4$.

The expression (4.3) derived in Section 4 for the right vertex function $\chi$, contributing where appropriate to the constant term in (1.5), represents a significant general term in the expansion of the spectral function (1.1) in the three-dimensional case, and courts confirmation within some more general differentialgeometric point of view. 


\section{Acknowledgements}

The author would like to thank the applied mathematicians at the University of Queensland, where most of this work was carried out during an Outside Studies Programme, for interesting and stimulating discussions concerning this subject.

\section{Appendix A: The circular limit}

In this appendix we use the preceding results to consider briefly the circular limit of a polygonal domain and the corresponding cylindrical region, with emphasis on the effects of the corner and vertex term contributions.

We first of all deal with the total corner angle term contribution $\alpha$, defined in (1.4), to the expansion (1.3) (two-dimensional case) for a regular polygon in the limit as the number of sides becomes infinite. If an internal angle in a regular $N$-gon is denoted by $\theta_{N}=\pi-2 \pi / N$, then $\theta_{N} \rightarrow \pi$ and

$$
N \alpha\left(\theta_{N}\right) \rightarrow \frac{1}{6} \text { as } N \rightarrow \infty \text {. }
$$

Thus for a circle this recovers the famous constant term 1/6 of Pleijel [9]. (A similar result was obtained by $\mathrm{Kac} \mathrm{[6]} \mathrm{in} \mathrm{considering} \mathrm{an} \mathrm{integral} \mathrm{representation} \mathrm{of}$ the corner angle term.)

Now consider the total vertex term contribution, defined in (4.3), to the expansion (1.5) (three-dimensional case) for a right regular polygonal prism end-piece, in the limit as the number of sides $N$ of the cross-section becomes infinite. If each right solid vertex angle so formed is denoted by $\Omega_{N}$, then $\Omega_{N} \rightarrow \pi$ and, by (4.3) and (A.1), we obtain, for the case of Dirichlet boundary conditions

$$
\begin{aligned}
-\operatorname{Lim}_{N \rightarrow \infty}\left\{N \chi\left(\Omega_{N}\right)\right\} & =-\frac{1}{4} \operatorname{Lim}_{N \rightarrow \infty}\left\{N \alpha\left(\Omega_{N}\right)\right\} \\
& =-\frac{1}{4} \cdot \frac{1}{6}=-\frac{1}{24} .
\end{aligned}
$$

The result (A.2) represents the contribution to the constant term in the expansion of the spectral function (1.1) in the three-dimensional case for a right circular cylindrical end-piece. This is confirmed by the particular calculations for cylinders wtih cross-section bounded by a smooth convex curve (via their Green's function approach) of Waechter [15] and Sleeman and Zayed [11], [12] which include the constant term $-1 / 12$ in the spectral function expansions (there being two end contributions from (A.2)). The sign change for the case of Neumann boundary conditions indicated in (1.5) is achieved also in the calculations for that case in [11], [12]. 


\section{Appendix B: Some summation formulae}

\section{Using the identity}

$$
\sum_{m=0}^{\infty} \sum_{n=0}^{m}=\sum_{n=0}^{\infty} \sum_{m=n}^{\infty}
$$

and the formula (c.f. [j], p. 14)

$$
\sum_{m=0}^{\infty} \sum_{n=0}^{\infty} f(n, m+n)=\sum_{m=0}^{\infty} \sum_{n=0}^{m} f(n, m)
$$

we deduce that

$$
\sum_{m=0}^{\infty} \sum_{n=0}^{\infty}[f(n, m+n)+f(m+n, n)]=\sum_{m=0}^{\infty} \sum_{n=0}^{\infty} f(m, n)+\sum_{m=0}^{\infty} f(m, m) .
$$

Thus if $f(m, n)$ is symmetric in its arguments, we have

$$
\sum_{m=0}^{\infty} \sum_{n=0}^{\infty} f(m+n, n)=\frac{1}{2}\left[\sum_{m=0}^{\infty} \sum_{n=0}^{\infty} f(m, n)+\sum_{m=0}^{\infty} f(m, m)\right] .
$$

Then, again if $f(m, n)=f(n, m)$, we find,

$$
\begin{aligned}
\sum_{m=1}^{\infty} & \sum_{n=1}^{\infty} f(m+n, n) \\
= & \frac{1}{2} \sum_{m=0}^{\infty} \sum_{n=0}^{\infty} f(m, n)-\frac{1}{2} \sum_{m=0}^{\infty} f(m, m)-\sum_{m=0}^{\infty} f(m, 0)+f(0,0),
\end{aligned}
$$

and

$$
\sum_{m=1}^{\infty} \sum_{n=0}^{\infty} f(m+n, n)=\frac{1}{2} \sum_{m=0}^{\infty} \sum_{n=0}^{\infty} f(m, n)-\frac{1}{2} \sum_{m=0}^{\infty} f(m, m)
$$

\section{References}

[1] H. P. Baltes, "Asymptotic eigenvalue distribution for the wave equation in a cylinder of arbitrary cross section", Phys. Rev. A 6 (1972) 2252-2257.

[2] J. Brossard and R. Carmona, "Can one hear the dimension of a fractal?", Comm. Math. Phys 104 (1986) 103-122.

[3] H. P. W. Gottlieb, "Hearing the shape of an annular drum", J. Austral. Math. Soc. Ser. B 24 (1983) $435-438$.

[4] H. P. W. Gottlieb, "Eigenvalues of the Laplacian with Neumann boundary conditions", $J$. Austral. Math. Soc. Ser. B 26 (1985) 293-309. 
[5] E. R. Hansen, $A$ table of series and products (Prentice-Hall, Englewood Cliffs, 1975).

[6] M. Kac, "Can one hear the shape of a drum?", Amer. Math. Monthly 73 (1966) 1-23.

[7] H. P. McKean and I. M. Singer, "Curvature and the eigenvalues of the Laplacian", $J$. Differential Geom. 1 (1967) 43-69.

[8] P. M. Morse and H. Feshbach, Methods of theoretical physics, Vol. 1 (McGraw-Hill, New York, 1953).

[9] A. Pleijel, "A study of certain Green's functions with applications in the theory of vibrating membranes", Ark. Mat. 2 (1953-1954) 553-569.

[10] B. D. Sleeman, "The inverse problem of acoustic scattering", IMA J. Appl. Math. 29 (1982) 113-142.

[11] B. D. Sleeman and E. M. E. Zayed, "An inverse eigenvalue problem for a general convex domain", J. Math. Anal. Appl. 94 (1983) 78-95.

[12] B. D. Sleeman and E. M. E. Zayed, "Trace formula for the eigenvalues of the Laplacian", ZAMP J. Appl. Math. Phys. 35 (1984) 106-115.

[13] K. Stewartson and R. T. Waechter, "On hearing the shape of a drum: further results", Proc. Cambridge Philos. Soc. 69 (1971) 353-363.

[14] H. Urakawa, "Bounded domains which are isospectral but not congruent", Ann. Sct. École Norm. Sup. 15 (1982) 441-456.

[15] R. T. Waechter, "On hearing the shape of a drum: an extension to higher dimensions", Proc. Cambridge Philos. Soc. 72 (1972) 439-447.

[16] E. M. E. Zayed, "An inverse eigenvalue problem for the Laplace operator", in Ordinary and partial differential equations, (eds. W. N. Everitt and B. D. Sleeman), (Springer, Berlin, 1982) $718-726$.

[17] E. M. E. Zayed, "Eigenvalues of the Laplacian: an extension to higher dimensions", IMA J. Appl. Math. 33 (1984) 83-99. 